Network Working Group

Request for Comments: 1490

Obsoletes: 1294
T. Bradley

Wellfleet Communications, Inc.

C. Brown

Wellfleet Communications, Inc.

A. Malis

Ascom Timeplex, Inc.

July 1993

Multiprotocol Interconnect over Frame Relay

Status of this Memo

This RFC specifies an IAB standards track protocol for the Internet community, and requests discussion and suggestions for improvements. Please refer to the current edition of the "IAB Official Protocol

Standards" for the standardization state and status of this protocol. Distribution of this memo is unlimited.

\title{
Abstract
}

This memo describes an encapsulation method for carrying network interconnect traffic over a Frame Relay backbone. It covers aspects of both Bridging and Routing. Additionally, it describes a simple fragmentation procedure for carrying large frames over a frame relay network with a smaller MTU.

Systems with the ability to transfer both the encapsulation method described in this document, and others must have a priori knowledge of which virtual circuits will carry which encapsulation method and this encapsulation must only be used over virtual circuits that have been explicitly configured for its use.

Acknowledgements

Comments and contributions from many sources, especially those from Ray Samora of Proteon, Ken Rehbehn of Netrix Corporation, Fred Baker and Charles Carvalho of Advanced Computer Communications and Mostafa Sherif of AT\&T have been incorporated into this document. Special thanks to Dory Leifer of University of Michigan for his contributions to the resolution of fragmentation issues and Floyd Backes from DEC and Laura Bridge from Timeplex for their contributions to the bridging descriptions. This document could not have been completed without the expertise of the IP over Large Public Data Networks working group of the IETF. 


\section{Conventions and Acronyms}

The following language conventions are used in the items of specification in this document:

- Must, Shall or Mandatory -- the item is an absolute requirement of the specification.

o Should or Recommended -- the item should generally be followed for all but exceptional circumstances.

- May or Optional -- the item is truly optional and may be followed or ignored according to the needs of the implementor.

All drawings in this document are drawn with the left-most bit as the high order bit for transmission. For example, the dawings might be labeled as:

$\begin{array}{lllllllll}0 & 1 & 2 & 3 & 4 & 5 & 6 & 7 & \text { bits }\end{array}$

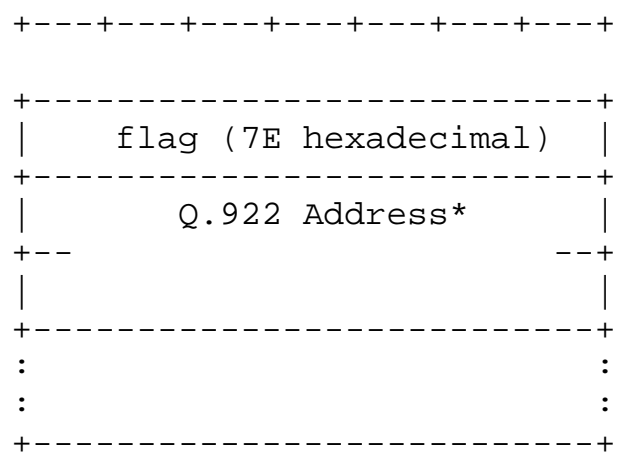

Drawings that would be too large to fit onto one page if each octet were presented on a single line are drawn with two octets per line. These are also drawn with the left-most bit as the high order bit for transmission. There will be a "+" to distinguish between octets as in the following example. 

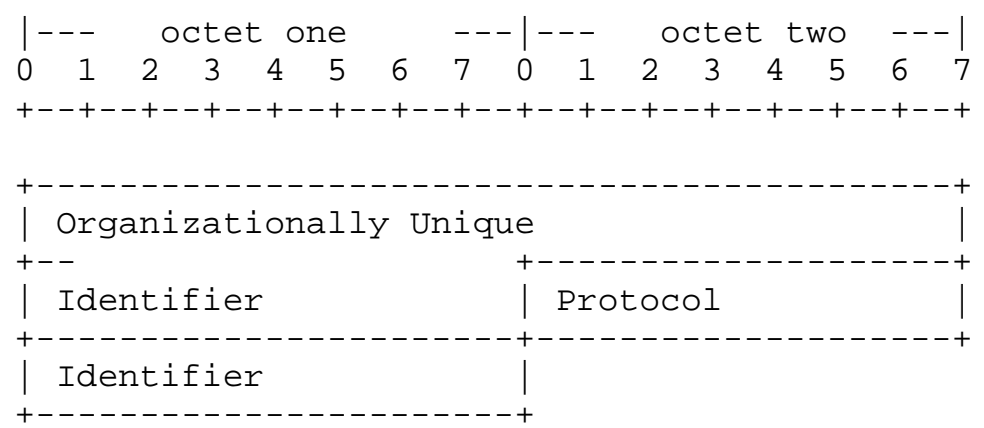

The following are common acronyms used throughout this document.

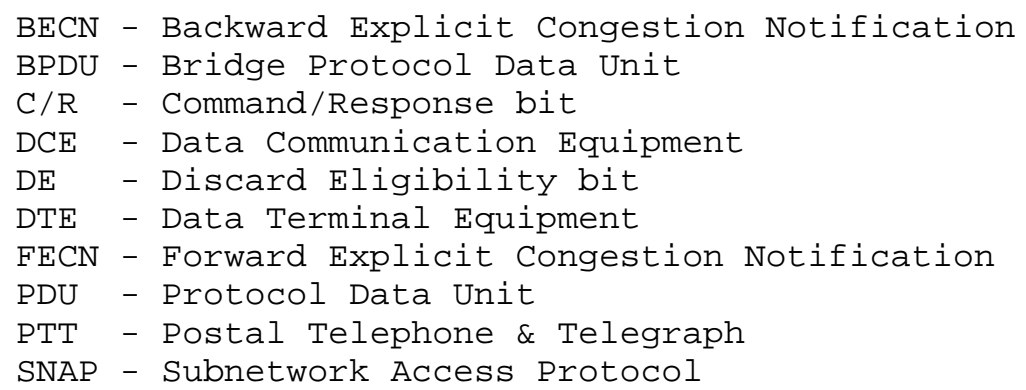

2. Introduction

The following discussion applies to those devices which serve as end stations (DTEs) on a public or private Frame Relay network (for example, provided by a common carrier or PTT. It will not discuss the behavior of those stations that are considered a part of the Frame Relay network (DCES) other than to explain situations in which the DTE must react.

The Frame Relay network provides a number of virtual circuits that form the basis for connections between stations attached to the same Frame Relay network. The resulting set of interconnected devices forms a private Frame Relay group which may be either fully interconnected with a complete "mesh" of virtual circuits, or only partially interconnected. In either case, each virtual circuit is uniquely identified at each Frame Relay interface by a Data Link Connection Identifier (DLCI). In most circumstances, DLCIs have strictly local significance at each Frame Relay interface.

The specifications in this document are intended to apply to both switched and permanent virtual circuits. 


\section{Frame Format}

All protocols must encapsulate their packets within a Q.922 Annex A frame [1,2]. Additionally, frames shall contain information necessary to identify the protocol carried within the protocol data unit (PDU), thus allowing the receiver to properly process the incoming packet. The format shall be as follows:

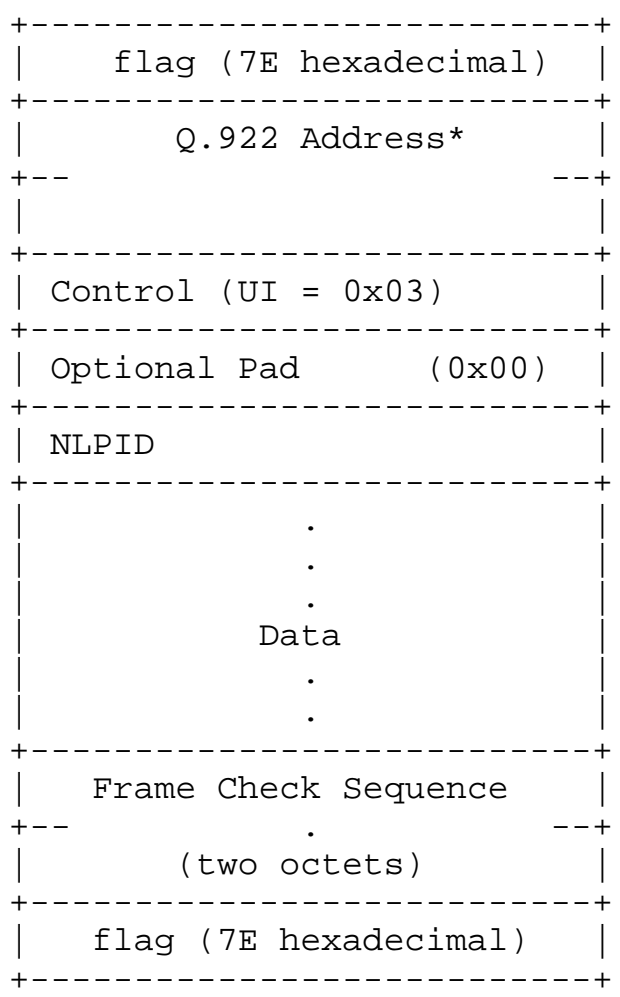

* Q.922 addresses, as presently defined, are two octets and contain a 10-bit DLCI. In some networks Q.922 addresses may optionally be increased to three or four octets.

The control field is the $Q .922$ control field. The UI $(0 \times 03)$ value is used unless it is negotiated otherwise. The use of XID (OxAF or $0 \times B F)$ is permitted and is discussed later.

The pad field is used to align the remainder of the frame to a two octet boundary. There may be zero or one pad octet within the pad field and, if present, must have a value of zero.

The Network Level Protocol ID (NLPID) field is administered by ISO 
and CCITT. It contains values for many different protocols including IP, CLNP and IEEE Subnetwork Access Protocol (SNAP)[10]. This field tells the receiver what encapsulation or what protocol follows. Values for this field are defined in ISO/IEC TR 9577 [3]. A NLPID value of $0 \times 00$ is defined within ISO/IEC TR 9577 as the Null Network Layer or Inactive set. Since it cannot be distinguished from a pad field, and because it has no significance within the context of this encapsulation scheme, a NLPID value of $0 \times 00$ is invalid under the Frame Relay encapsulation. The Appendix contains a list of some of the more commonly used NLPID values.

There is no commonly implemented minimum maximum frame size for Frame Relay. A network must, however, support at least a 262 octet maximum. Generally, the maximum will be greater than or equal to 1600 octets, but each Frame Relay provider will specify an appropriate value for its network. A Frame Relay DTE, therefore, must allow the maximum acceptable frame size to be configurable.

The minimum frame size allowed for Frame Relay is five octets between the opening and closing flags assuming a two octet $Q .922$ address field. This minimum increases to six octets for three octet Q.922 address and seven octets for the four octet Q.922 address format.

\section{Interconnect Issues}

There are two basic types of data packets that travel within the Frame Relay network: routed packets and bridged packets. These packets have distinct formats and therefore, must contain an indicator that the destination may use to correctly interpret the contents of the frame. This indicator is embedded within the NLPID and SNAP header information.

For those protocols that do not have a NLPID already assigned, it is necessary to provide a mechanism to allow easy protocol identification. There is a NLPID value defined indicating the presence of a SNAP header.

A SNAP header is of the form:

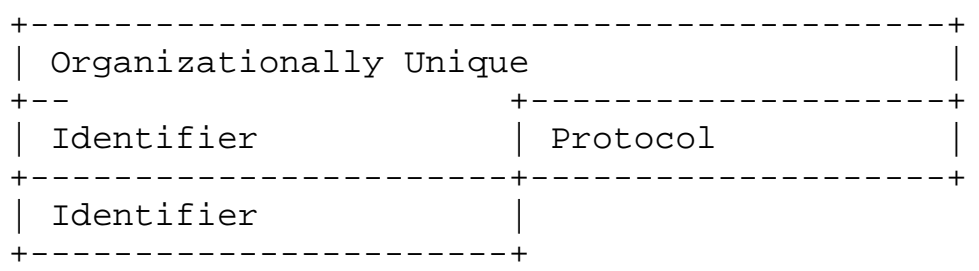

All stations must be able to accept and properly interpret both the 
NLPID encapsulation and the SNAP header encapsulation for a routed packet.

The three-octet Organizationally Unique Identifier (OUI) identifies an organization which administers the meaning of the Protocol Identifier (PID) which follows. Together they identify a distinct protocol. Note that OUI 0x00-00-00 specifies that the following PID is an Ethertype.

\subsection{Routed Frames}

Some protocols will have an assigned NLPID, but because the NLPID numbering space is so limited, not all protocols have specific NLPID values assigned to them. When packets of such protocols are routed over Frame Relay networks, they are sent using the NLPID 0x80 (which indicates a SNAP follows) followed by SNAP. If the protocol has an Ethertype assigned, the OUI is 0x00-00-00 (which indicates an Ethertype follows), and PID is the Ethertype of the protocol in use. There will be one pad octet to align the protocol data on a two octet boundary as shown below.

Format of Routed Frames

with Ethertypes

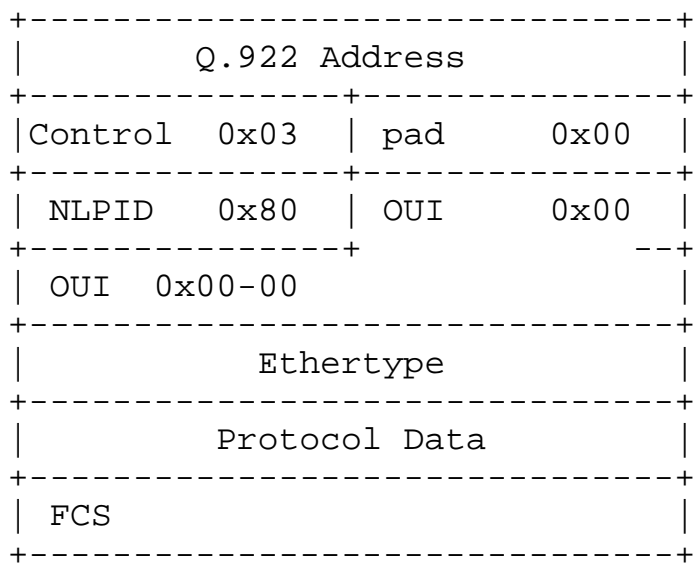

In the few cases when a protocol has an assigned NLPID (see appendix), 48 bits can be saved using the format below: 
Format of Routed NLPID Protocol

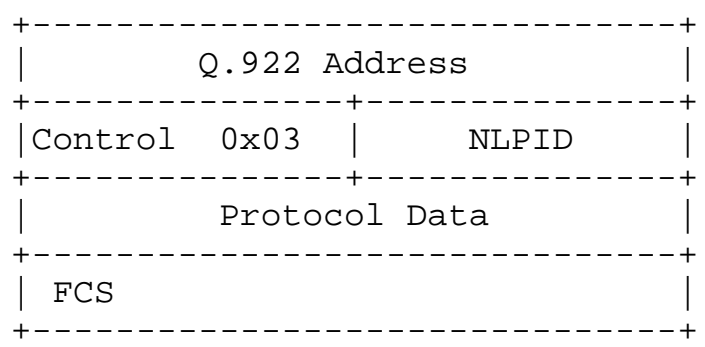

The NLPID encapsulation does not require a pad octet for alignment, so none is permitted.

In the case of ISO protocols, the NLPID is considered to be the first octet of the protocol data. It is unnecessary to repeat the NLPID in this case. The single octet serves both as the demultiplexing value and as part of the protocol data (refer to "Other Protocols over Frame Relay for more details). Other protocols, such as IP, have a NLPID defined $(0 x C C)$, but it is not part of the protocol itself.

Format of Routed IP Datagram
Q.922 Address
+-------------+-------------+
| Control $0 \times 03$ | NLPID $0 \times C C$
+------------+------------+
IP Datagram
+ FCS

\subsection{Bridged Frames}

The second type of Frame Relay traffic is bridged packets. These packets are encapsulated using the NLPID value of 0x80 indicating SNAP. As with other SNAP encapsulated protocols, there will be one pad octet to align the data portion of the encapsulated frame. The SNAP header which follows the NLPID identifies the format of the bridged packet. The OUI value used for this encapsulation is the 802.1 organization code 0x00-80-C2. The PID portion of the SNAP header (the two bytes immediately following the OUI) specifies the form of the MAC header, which immediately follows the SNAP header. Additionally, the PID indicates whether the original FCS is preserved within the bridged frame.

The 802.1 organization has reserved the following values to be used with Frame Relay: 


\begin{tabular}{lll}
\multicolumn{1}{c}{ PID Values for OUI $0 \times 00-80-\mathrm{C} 2$} & \\
with preserved FCS & w/O preserved FCS & Media \\
------------- & -------------- & -------------- \\
$0 \times 00-01$ & $0 \times 00-07$ & $802.3 /$ Ethernet \\
$0 \times 00-02$ & $0 \times 00-08$ & 802.4 \\
$0 \times 00-03$ & $0 \times 00-09$ & 802.5 \\
$0 \times 00-04$ & $0 \times 00-0 \mathrm{~A}$ & FDDI \\
& $0 \times 00-0 \mathrm{~B}$ & 802.6
\end{tabular}

In addition, the PID value 0x00-0E, when used with OUI 0x00-80-C2, identifies bridged protocol data units (BPDUs) as defined by $802.1(\mathrm{~d})$ or $802.1(\mathrm{~g})$ [12].

A packet bridged over Frame Relay will, therefore, have one of the following formats:

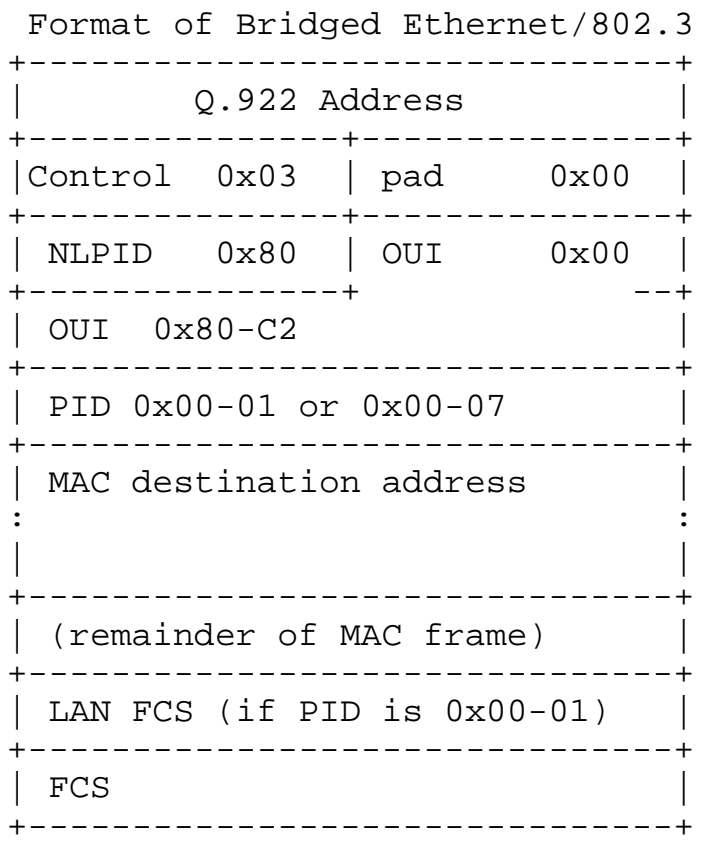


Format of Bridged 802.4 Frame

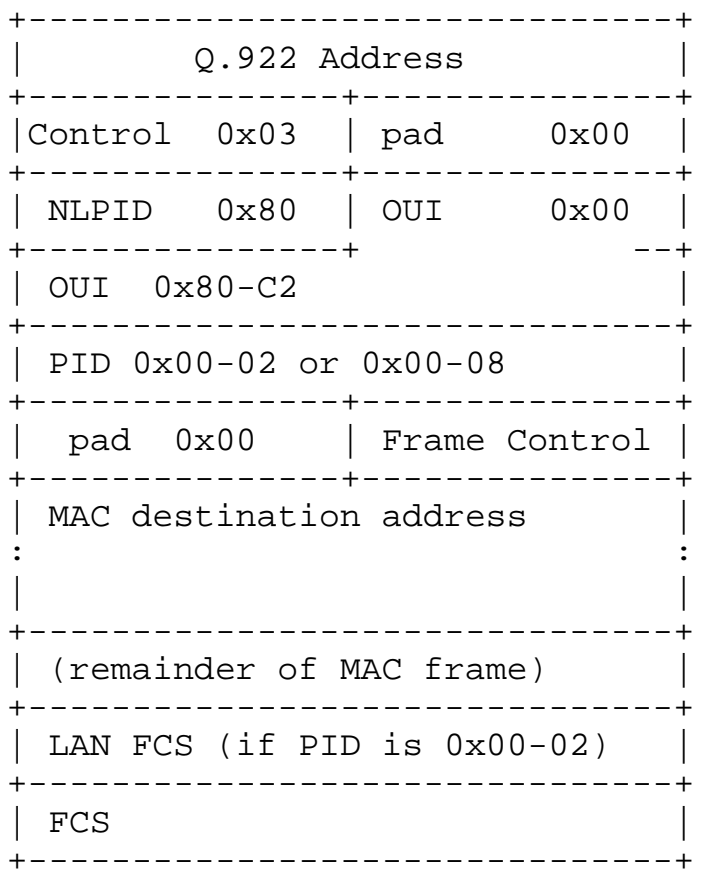


Format of Bridged 802.5 Frame

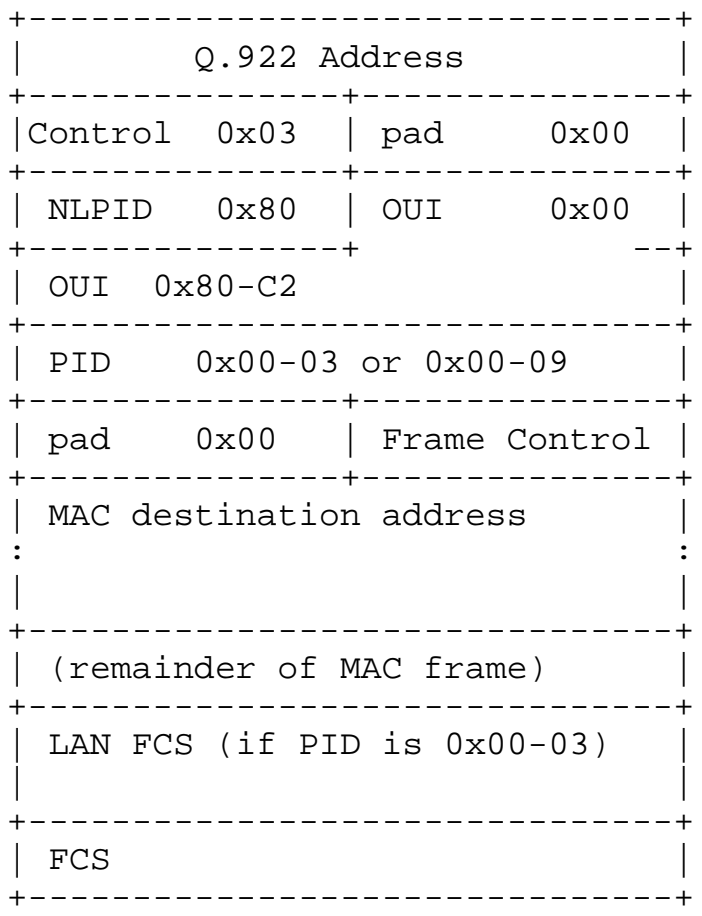




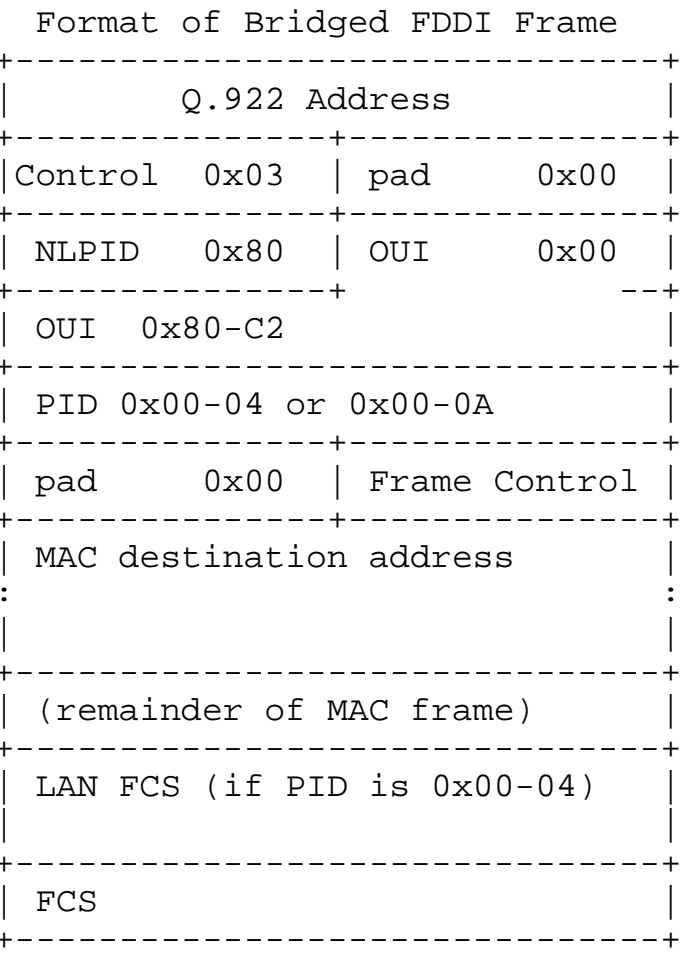




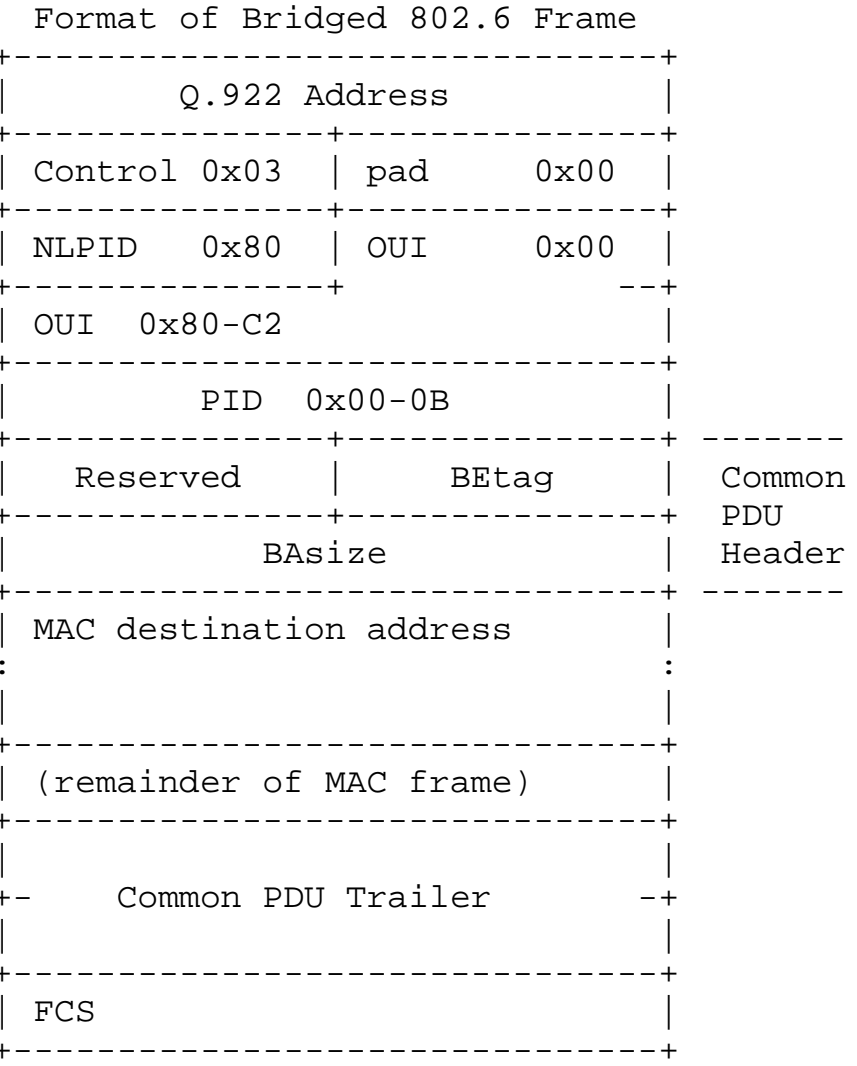

Note that in bridge 802.6 PDUs, there is only one choice for the PID value, since the presence of a CRC-32 is indicated by the CIB bit in the header of the MAC frame.

The Common Protocol Data Unit (CPDU) Header and Trailer are conveyed to allow pipelining at the egress bridge to an 802.6 subnetwork. Specifically, the CPDU Header contains the BAsize field, which contains the length of the PDU. If this field is not available to the egress 802.6 bridge, then that bridge cannot begin to transmit the segmented PDU until it has received the entire PDU, calculated the length, and inserted the length into the BAsize field. If the field is available, the egress 802.6 bridge can extract the length from the BAsize field of the Common PDU Header, insert it into the corresponding field of the first segment, and immediately transmit the segment onto the 802.6 subnetwork. Thus, the bridge can begin transmitting the 802.6 PDU before it has received the complete PDU.

One should note that the Common PDU Header and Trailer of the encapsulated frame should not be simply copied to the outgoing 802.6 
subnetwork because the encapsulated BEtag value may conflict with the previous BEtag value transmitted by that bridge.

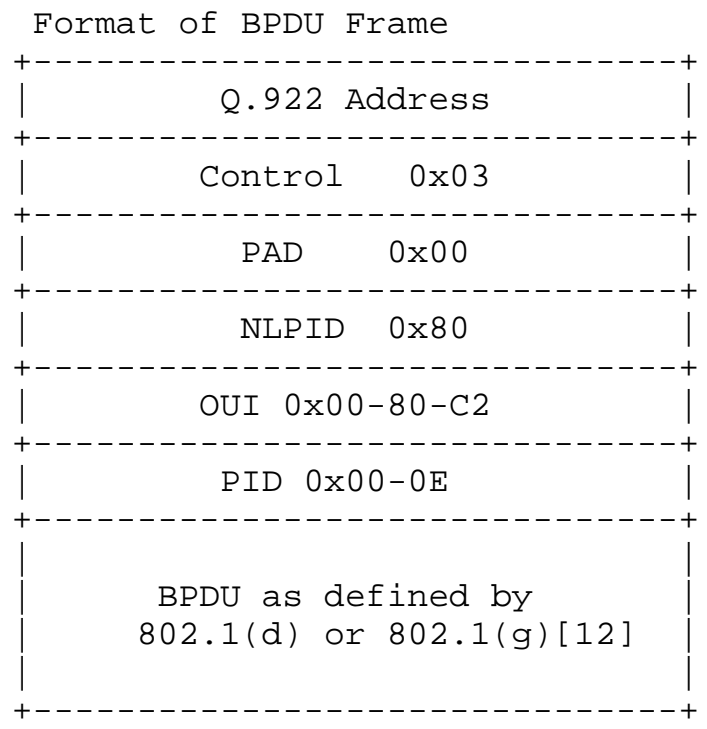

4. Data Link Layer Parameter Negotiation

Frame Relay stations may choose to support the Exchange

Identification (XID) specified in Appendix III of Q.922 [1]. This XID exchange allows the following parameters to be negotiated at the initialization of a Frame Relay circuit: maximum frame size N201, retransmission timer T200, and the maximum number of outstanding Information (I) frames $\mathrm{K}$.

A station may indicate its unwillingness to support acknowledged mode multiple frame operation by specifying a value of zero for the maximum window size, $\mathrm{K}$.

If this exchange is not used, these values must be statically configured by mutual agreement of Data Link Connection (DLC) endpoints, or must be defaulted to the values specified in Section 5.9 of Q.922: 


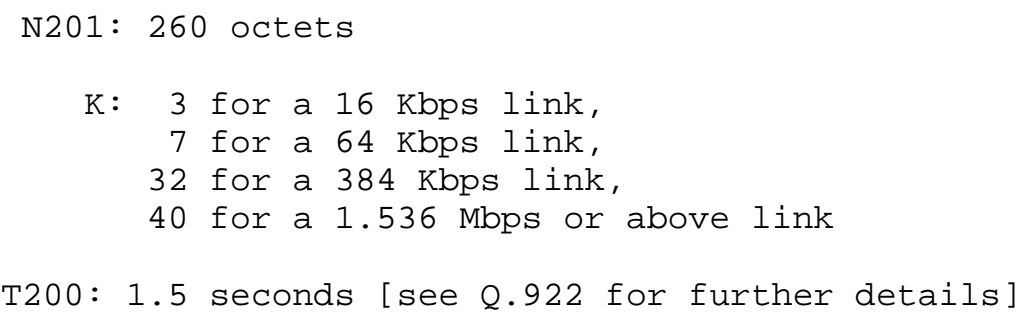

If a station supporting XID receives an XID frame, it shall respond with an XID response. In processing an XID, if the remote maximum frame size is smaller than the local maximum, the local system shall reduce the maximum size it uses over this DLC to the remotely specified value. Note that this shall be done before generating a response XID.

The following diagram describes the use of XID to specify non-use of acknowledged mode multiple frame operation. 


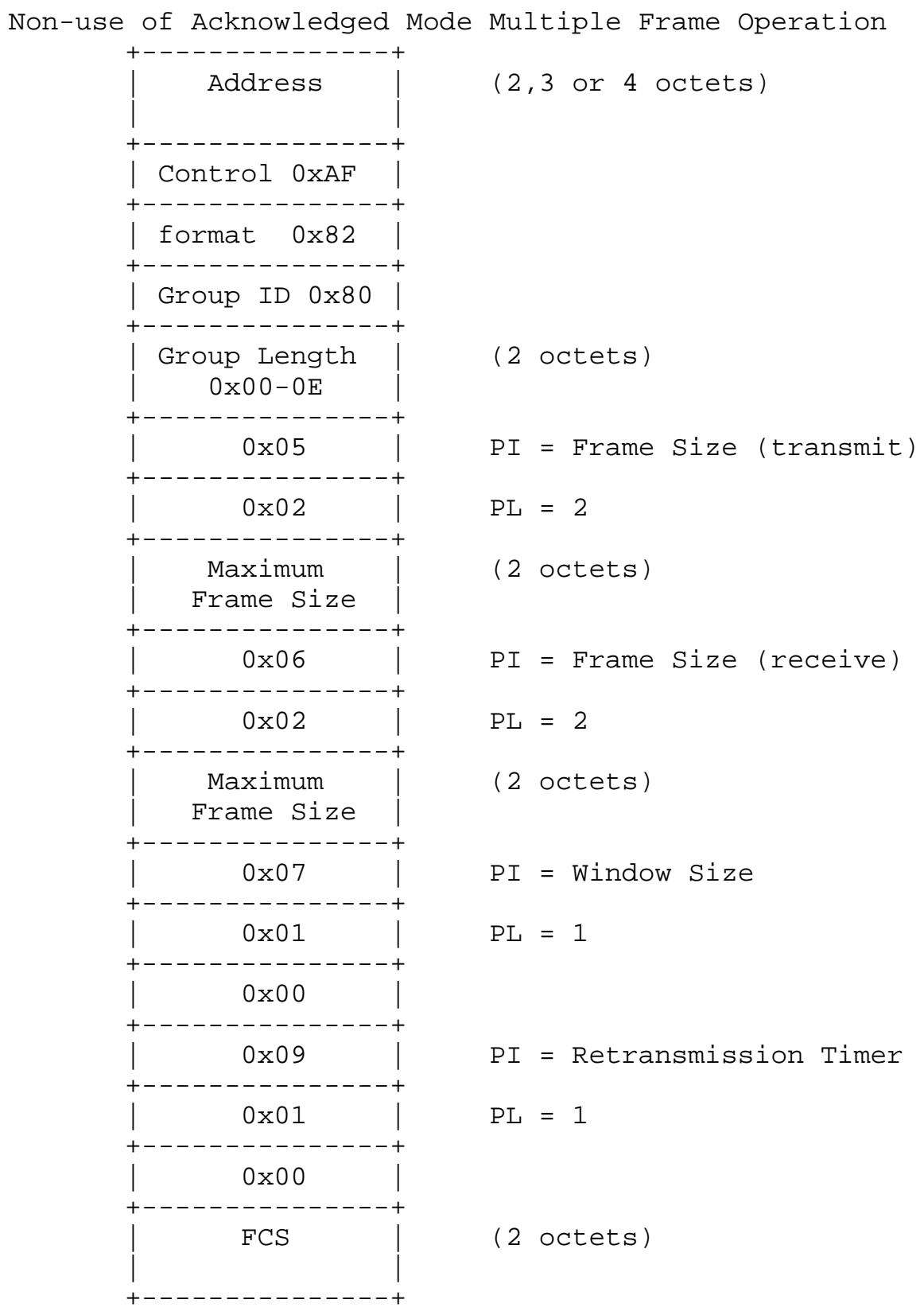

6. Fragmentation Issues

Fragmentation allows the exchange of packets that are greater than the maximum frame size supported by the underlying network. In the 
case of Frame Relay, the network may support a maximum frame size as small as 262 octets. Because of this small maximum size, it is recommended, but not required, to support fragmentation and reassembly.

Unlike IP fragmentation procedures, the scope of Frame Relay fragmentation procedure is limited to the boundary (or DTEs) of the Frame Relay network.

The general format of fragmented packets is the same as any other encapsulated protocol. The most significant difference being that the fragmented packet will contain the encapsulation header. That is, a packet is first encapsulated (with the exception of the address and control fields) as defined above. Large packets are then broken up into frames appropriate for the given Frame Relay network and are encapsulated using the Frame Relay fragmentation format. In this way, a station receiving fragments may reassemble them and then put the reassembled packet through the same processing path as a packet that had not been fragmented.

Within Frame Relay fragments are encapsulated using the SNAP format with an OUI of 0x00-80-C2 and a PID of 0x00-0D. Individual fragments will, therefore, have the following format:

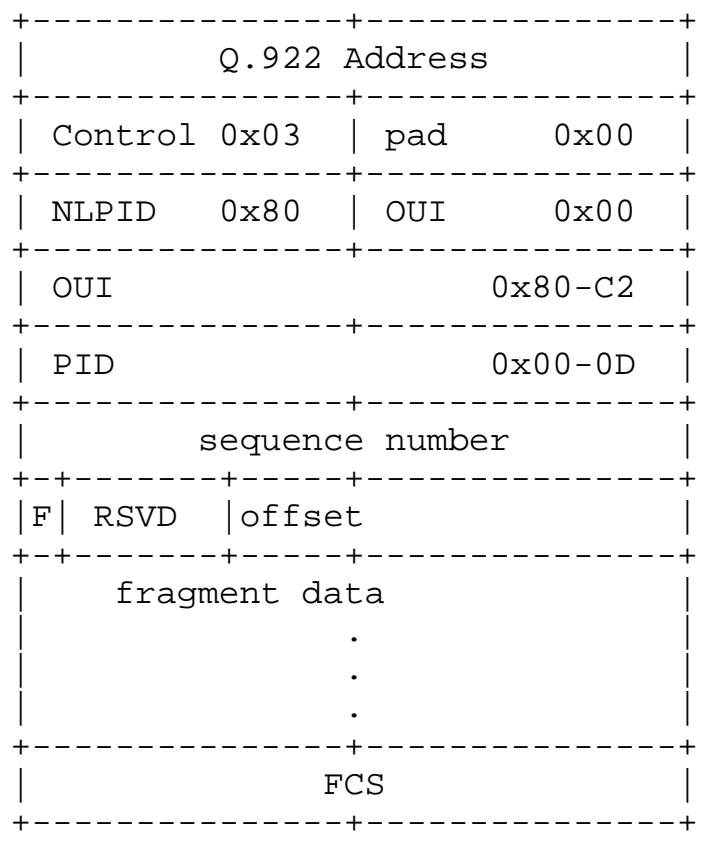

The sequence field is a two octet identifier that is incremented 
every time a new complete message is fragmented. It allows detection of lost frames and is set to a random value at initialization.

The reserved field is 4 bits long and is not currently defined. It must be set to 0 .

The final bit is a one bit field set to 1 on the last fragment and set to 0 for all other fragments.

The offset field is an 11 bit value representing the logical offset of this fragment in bytes divided by 32 . The first fragment must have an offset of zero.

The following figure shows how a large IP datagram is fragmented over Frame Relay. In this example, the complete datagram is fragmented into two Frame Relay frames. 


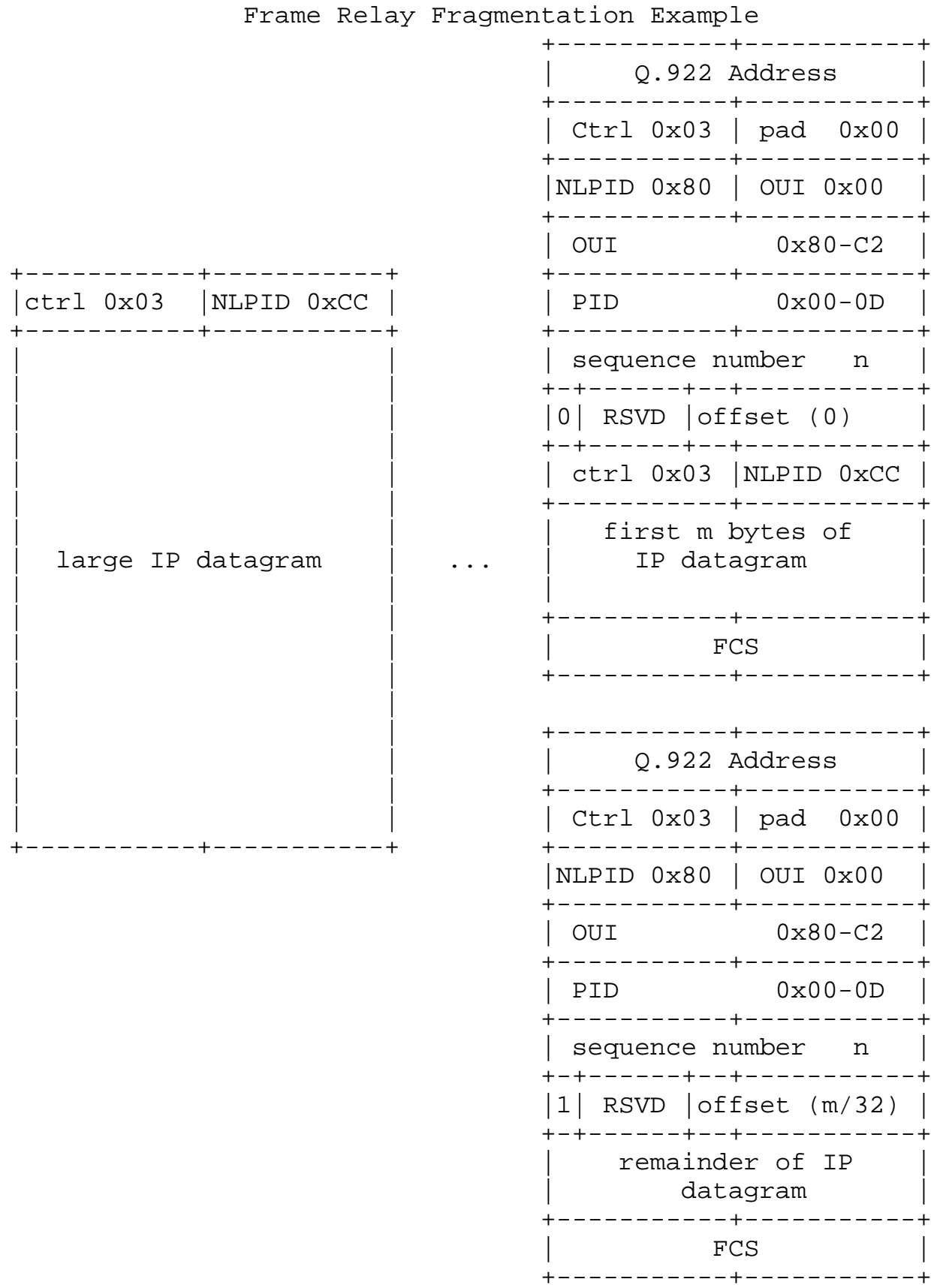

Fragmentation Example

Fragments must be sent in order starting with a zero offset and ending with the final fragment. These fragments must not be 
interrupted with other packets or information intended for the same DLC. An end station must be able to re-assemble up to $2 \mathrm{~K}$ octets and is suggested to support up to $8 \mathrm{~K}$ octet re-assembly. If at any time during this re-assembly process, a fragment is corrupted or a fragment is missing, the entire message is dropped. The upper layer protocol is responsible for any retransmission in this case. Note that there is no reassembly timer, nor is one needed. This is because the Frame Relay service is required to deliver frames in order.

This fragmentation algorithm is not intended to reliably handle all possible failure conditions. As with IP fragmentation, there is a small possibility of reassembly error and delivery of an erroneous packet. Inclusion of a higher layer checksum greatly reduces this risk.

\section{Address Resolution}

There are situations in which a Frame Relay station may wish to dynamically resolve a protocol address. Address resolution may be accomplished using the standard Address Resolution Protocol (ARP) [6] encapsulated within a SNAP encoded Frame Relay packet as follows:

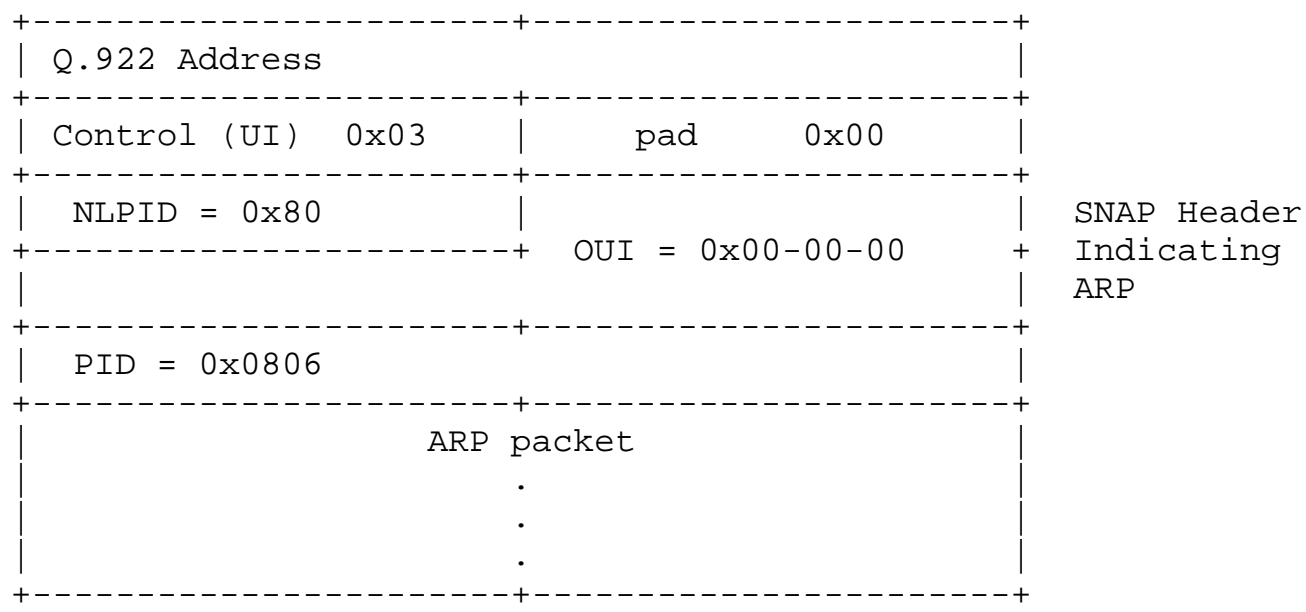

Where the ARP packet has the following format and values:

Data:

$\begin{array}{lr}\text { ar\$hrd } & 16 \text { bits } \\ \text { ar\$pro } & 16 \text { bits } \\ \text { ar\$hln } & 8 \text { bits }\end{array}$

Hardware type

Protocol type

Octet length of hardware address ( $\mathrm{n}$ ) 


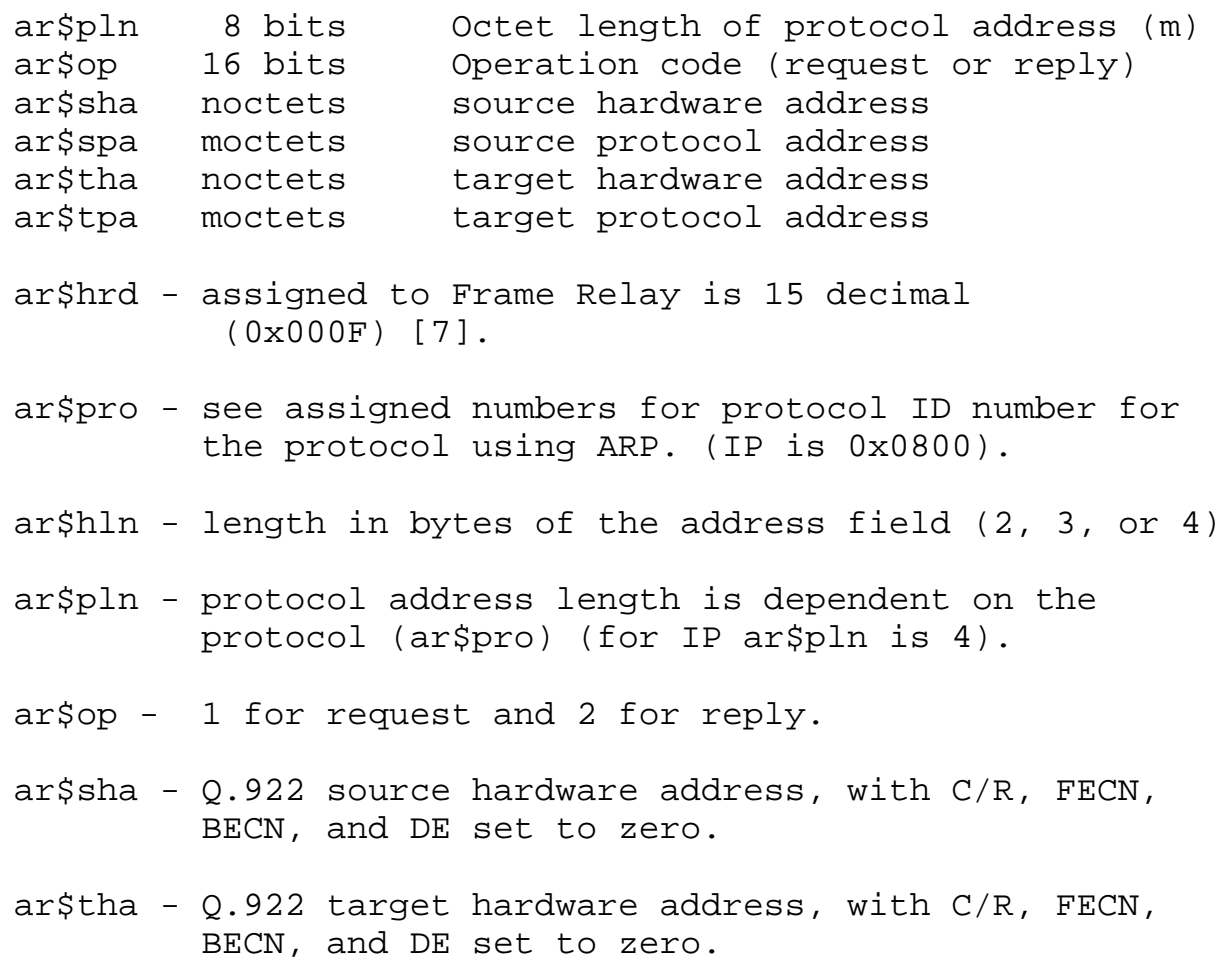

Because DLCIs within most Frame Relay networks have only local significance, an end station will not have a specific DLCI assigned to itself. Therefore, such a station does not have an address to put into the ARP request or reply. Fortunately, the Frame Relay network does provide a method for obtaining the correct DLCIs. The solution proposed for the locally addressed Frame Relay network below will work equally well for a network where DLCIs have global significance.

The DLCI carried within the Frame Relay header is modified as it traverses the network. When the packet arrives at its destination, the DLCI has been set to the value that, from the standpoint of the receiving station, corresponds to the sending station. For example, in figure 1 below, if station $A$ were to send a message to station $B$, it would place DLCI 50 in the Frame Relay header. When station $B$ received this message, however, the DLCI would have been modified by the network and would appear to B as DLCI 70 . 


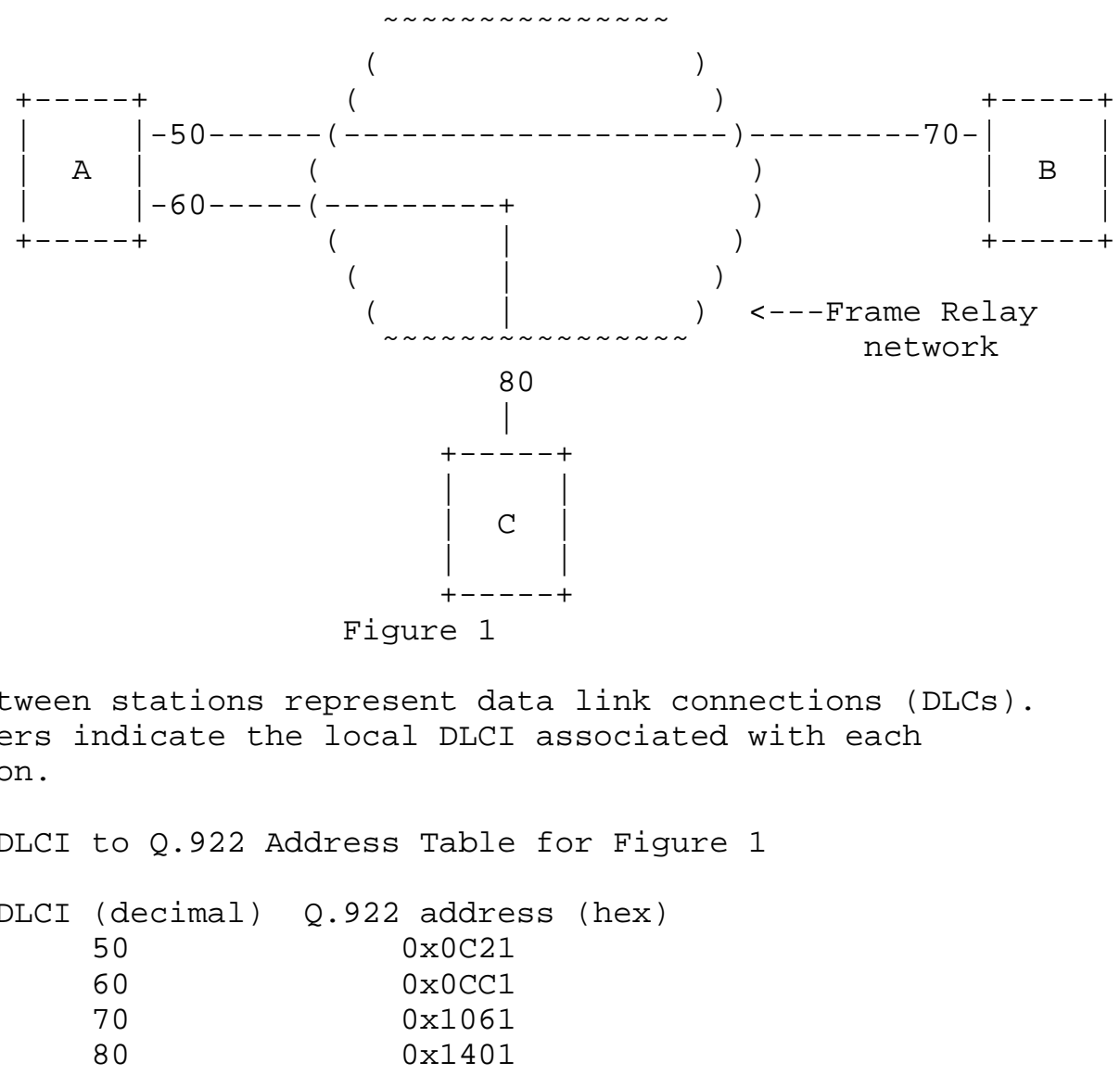

If you know about frame relay, you should understand the correlation between DLCI and Q.922 address. For the uninitiated, the translation between DLCI and Q.922 address is based on a two byte address length using the $\mathrm{Q} .922$ encoding format. The format is:

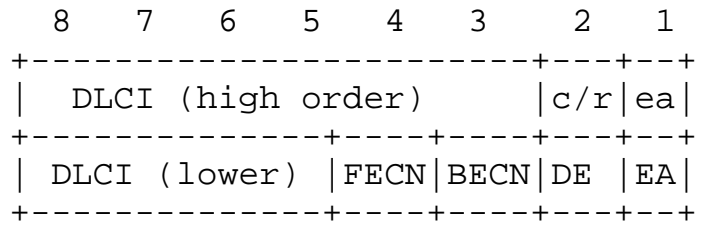

For ARP and its variants, the FECN, BECN, C/R and DE bits are assumed to be 0 .

When an ARP message reaches a destination, all hardware addresses 
will be invalid. The address found in the frame header will, however, be correct. Though it does violate the purity of layering, Frame Relay may use the address in the header as the sender hardware address. It should also be noted that the target hardware address, in both ARP request and reply, will also be invalid. This should not cause problems since ARP does not rely on these fields and in fact, an implementation may zero fill or ignore the target hardware address field entirely.

As an example of how this address replacement scheme may work, refer to figure 1. If station A (protocol address $\mathrm{pA}$ ) wished to resolve the address of station B (protocol address pB), it would format an ARP request with the following values:

$\begin{array}{cl}\text { ARP request } & \text { from } A \\ \text { ar\$op } & 1 \text { (request) } \\ \text { ar\$sha } & \text { unknown } \\ \text { ar\$spa } & \text { pA } \\ \text { ar\$tha } & \text { undefined } \\ \text { ar\$tpa } & \text { pB }\end{array}$

Because station A will not have a source address associated with it, the source hardware address field is not valid. Therefore, when the ARP packet is received, it must extract the correct address from the Frame Relay header and place it in the source hardware address field. This way, the ARP request from A will become:

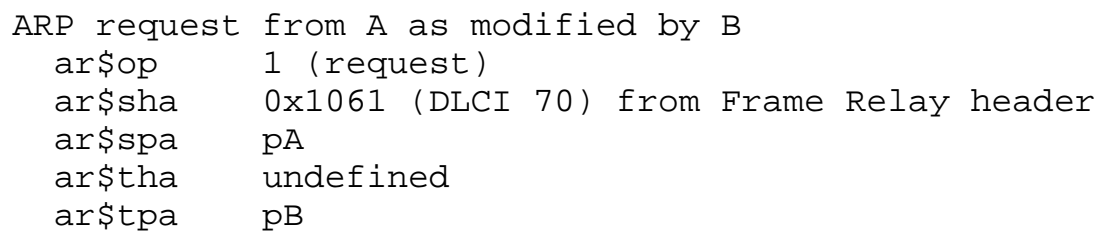

Station $B^{\prime}$ S ARP will then be able to store station A's protocol address and Q.922 address association correctly. Next, station B will form a reply message. Many implementations simply place the source addresses from the ARP request into the target addresses and then fills in the source addresses with its addresses. In this case, the ARP response would be:

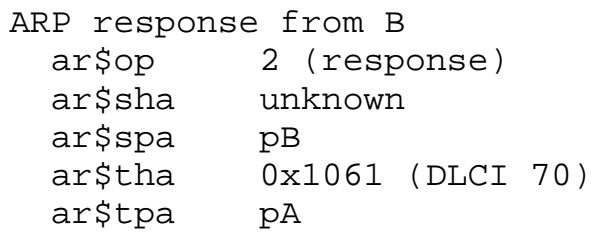


addressing may become useful in sending ARP requests and other "broadcast" messages.

Because of the inefficiencies of broadcasting in a Frame Relay environment, a new address resolution variation was developed. It is called Inverse ARP [11] and describes a method for resolving a protocol address when the hardware address is already known. In Frame Relay's case, the known hardware address is the DLCI. Using Inverse ARP for Frame Relay follows the same pattern as ARP and RARP use. That is the source hardware address is inserted at the receiving station.

In our example, station A may use Inverse ARP to discover the protocol address of the station associated with its DLCI 50 . The Inverse ARP request would be as follows:

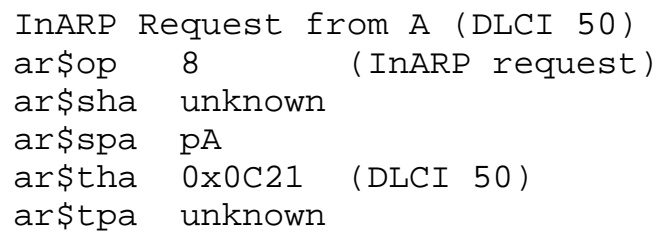

When station B receives this packet, it will modify the source hardware address with the Q.922 address from the Frame Relay header. This way, the InARP request from A will become:

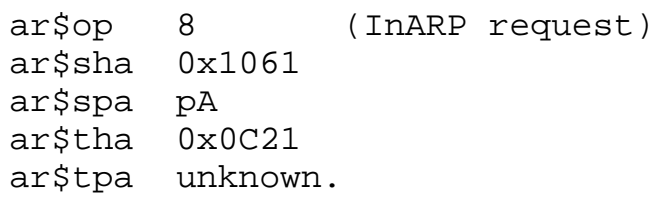

Station B will format an Inverse ARP response and send it to station $A$ as it would for any ARP message.

8. IP over Frame Relay

Internet Protocol [9] (IP) datagrams sent over a Frame Relay network conform to the encapsulation described previously. Within this context, IP could be encapsulated in two different ways. 
1. NLPID value indicating IP

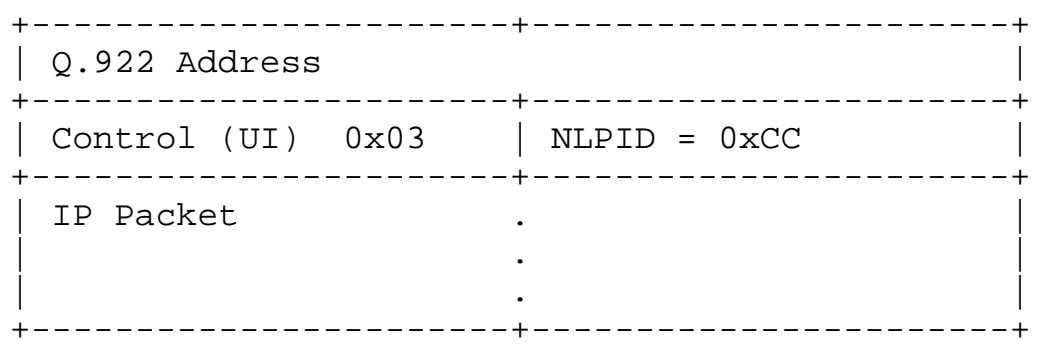

2. NLPID value indicating SNAP

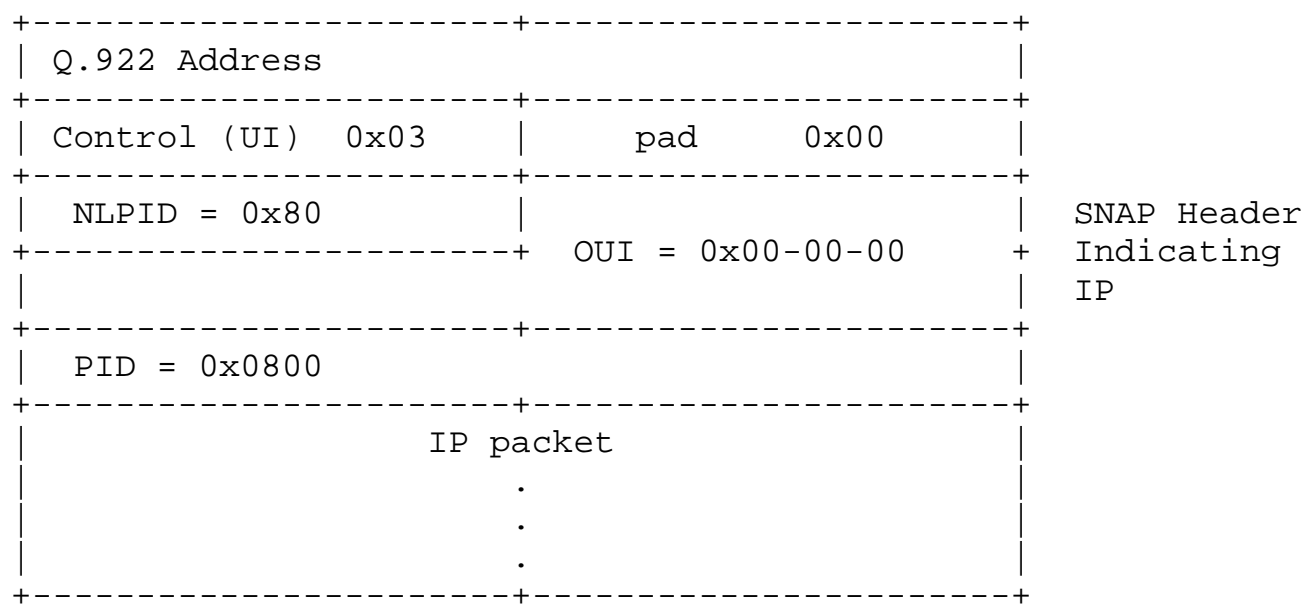

Although both of these encapsulations are supported under the given definitions, it is advantageous to select only one method as the appropriate mechanism for encapsulating IP data. Therefore, IP data shall be encapsulated using the NLPID value of 0xCC indicating IP as shown in option 1 above. This (option 1) is more efficient in transmission (48 fewer bits), and is consistent with the encapsulation of IP in X.25.

9. Other Protocols over Frame Relay

As with IP encapsulation, there are alternate ways to transmit various protocols within the scope of this definition. To eliminate the conflicts, the SNAP encapsulation is only used if no NLPID value is defined for the given protocol.

As an example of how this works, ISO CLNP has a NLPID defined (0x81). 
Therefore, the NLPID field will indicate ISO CLNP and the data packet will follow immediately. The frame would be as follows:

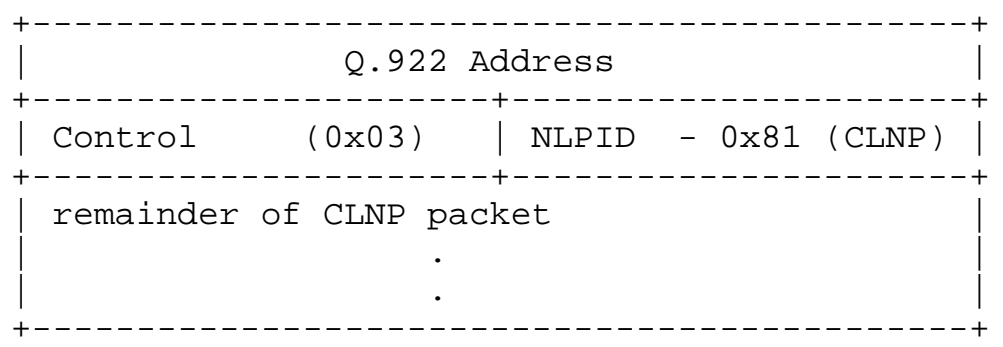

In this example, the NLPID is used to identify the data packet as CLNP. It is also considered part of the CLNP packet and as such, the NLPID should not be removed before being sent to the upper layers for processing. The NLPID is not duplicated.

Other protocols, such as IPX, do not have a NLPID value defined. As mentioned above, IPX would be encapsulated using the SNAP header. In this case, the frame would be as follows:

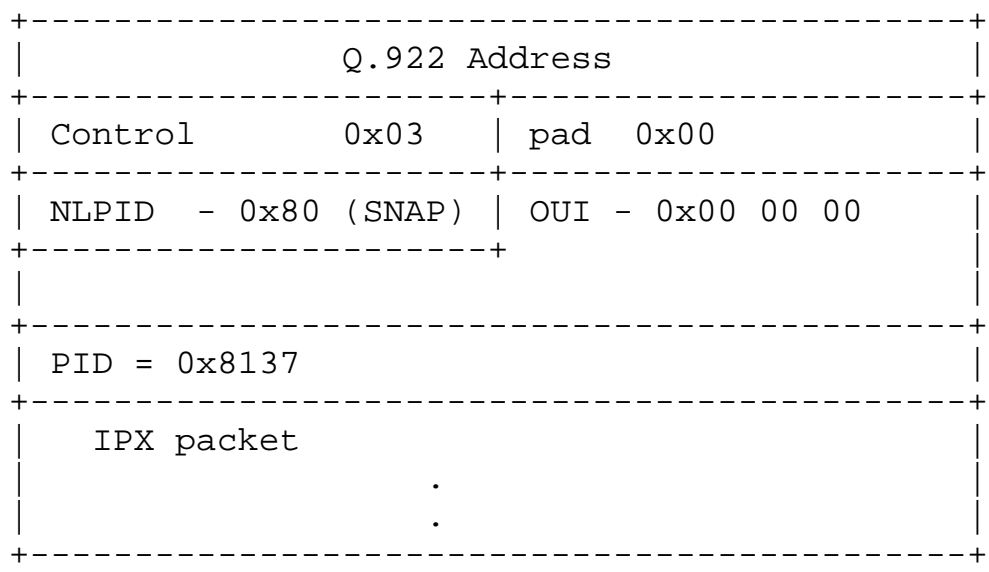

10. Bridging Model for Frame Relay

The model for bridging in a Frame Relay network is identical to the model for remote bridging as described in IEEE P802.1g "Remote MAC Bridging" [13] and supports the concept of "Virtual Ports". Remote bridges with LAN ports receive and transmit MAC frames to and from the LANS to which they are attached. They may also receive and transmit MAC frames through virtual ports to and from other remote bridges. A virtual port may represent an abstraction of a remote bridge's point of access to one, two or more other remote bridges. 
Remote Bridges are statically configured as members of a remote bridge group by management. All members of a remote bridge group are connected by one or more virtual ports. The set of remote MAC bridges in a remote bridge group provides actual or *potential* MAC layer interconnection between a set of LANs and other remote bridge groups to which the remote bridges attach.

In a Frame Relay network there must be a full mesh of Frame Relay VCs between bridges of a remote bridge group. If the frame relay network is not a full mesh, then the bridge network must be divided into multiple remote bridge groups.

The frame relay VCs that interconnect the bridges of a remote bridge group may be combined or used individually to form one or more virtual bridge ports. This gives flexibility to treat the Frame Relay interface either as a single virtual bridge port, with all VCs in a group, or as a collection of bridge ports (individual or grouped $\mathrm{VCs}$ ).

When a single virtual bridge port provides the interconnectivity for all bridges of a given remote bridge group (i.e. all VCs are combined into a single virtual port), the standard Spanning Tree Algorithm may be used to determine the state of the virtual port. When more than one virtual port is configured within a given remote bridge group then an "extended" Spanning Tree Algorithm is required. Such an extended algorithm is defined in IEEE 802.1g [13]. The operation of this algorithm is such that a virtual port is only put into backup if there is a loop in the network external to the remote bridge group.

The simplest bridge configuration for a Frame Relay network is the LAN view where all VCs are combined into a single virtual port. Frames, such as BPDUs, which would be broadcast on a LAN, must be flooded to each VC (or multicast if the service is developed for Frame Relay services). Flooding is performed by sending the packet to each relevant DLC associated with the Frame Relay interface. The VCs in this environment are generally invisible to the bridge. That is, the bridge sends a flooded frame to the frame relay interface and does not "see" that the frame is being forwarded to each VC individually. If all participating bridges are fully connected (full mesh) the standard Spanning Tree Algorithm will suffice in this configuration.

Typically LAN bridges learn which interface a particular end station may be reached on by associating a MAC address with a bridge port. In a Frame Relay network configured for the LAN-like single bridge port (or any set of VCs grouped together to form a single bridge port), however, the bridge must not only associated a MAC address with a bridge port, but it must also associate it with a connection 
identifier. For Frame Relay networks, this connection identifier is a DLCI. It is unreasonable and perhaps impossible to require bridges to statically configure an association of every possible destination MAC address with a DLC. Therefore, Frame Relay LAN-modeled bridges must provide a mechanism to allow the Frame Relay bridge port to dynamically learn the associations. To accomplish this dynamic learning, a bridged packet shall conform to the encapsulation described within section 7. In this way, the receiving Frame Relay interface will know to look into the bridged packet to gather the appropriate information.

A second Frame Relay bridging approach, the point-to-point view, treats each Frame Relay VC as a separate bridge port. Flooding and forwarding packets are significantly less complicated using the point-to-point approach because each bridge port has only one destination. There is no need to perform artificial flooding or to associate DLCIs with destination MAC addresses. Depending upon the interconnection of the VCs, an extended Spanning Tree algorithm may be required to permit all virtual ports to remain active as long as there are no true loops in the topology external to the remote bridge group.

It is also possible to combine the LAN view and the point-to-point view on a single Frame Relay interface. To do this, certain VCs are combined to form a single virtual bridge port while other VCs are independent bridge ports.

The following drawing illustrates the different possible bridging configurations. The dashed lines between boxes represent virtual circuits.

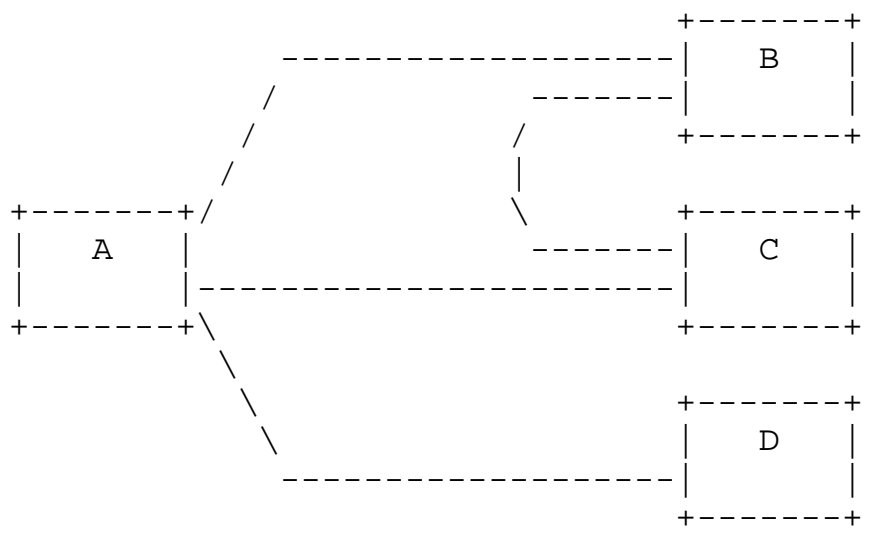

Since there is less than a full mesh of VCs between the bridges in this example, the network must be divided into more than one remote 
bridge group. A reasonable configuration is to have bridges A, B, and $\mathrm{C}$ in one group, and have bridges $\mathrm{A}$ and $\mathrm{D}$ in a second.

Configuration of the first bridge group combines the VCs interconnection the three bridges ( $A, B$, and $C$ ) into a single virtual port. This is an example of the LAN view configuration. The second group would also be a single virtual port which simply connects bridges $A$ and D. In this configuration the standard Spanning Tree Algorithm is sufficient to detect loops.

An alternative configuration has three individual virtual ports in the first group corresponding to the VCs interconnecting bridges A, B and $C$. Since the application of the standard Spanning Tree Algorithm to this configuration would detect a loop in the topology, an extended Spanning Tree Algorithm would have to be used in order for all virtual ports to be kept active. Note that the second group would still consist of a single virtual port and the standard Spanning Tree Algorithm could be used in this group.

Using the same drawing, one could construct a remote bridge scenario with three bridge groups. This would be an example of the point-topoint case. Here, the VC connecting A and B, the VC connecting A and $C$, and the VC connecting $A$ and $D$ are all bridge groups with a single virtual port. 
11. Appendix A

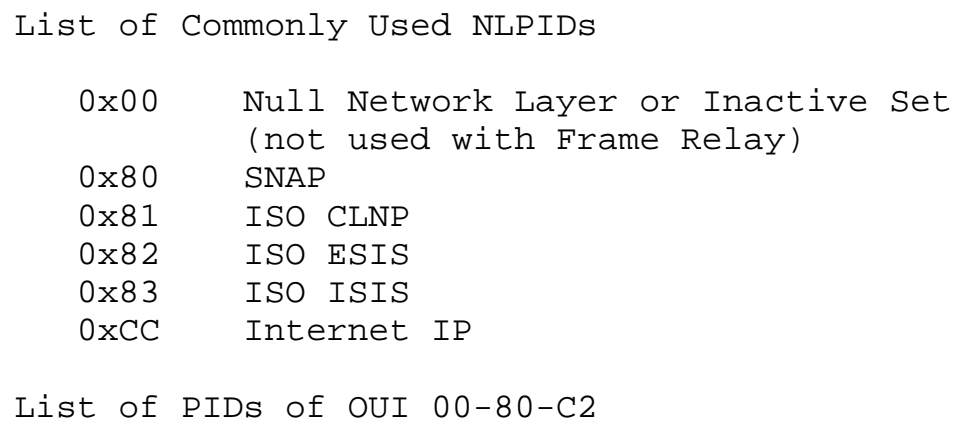

12. Appendix B - Connection Oriented procedures.

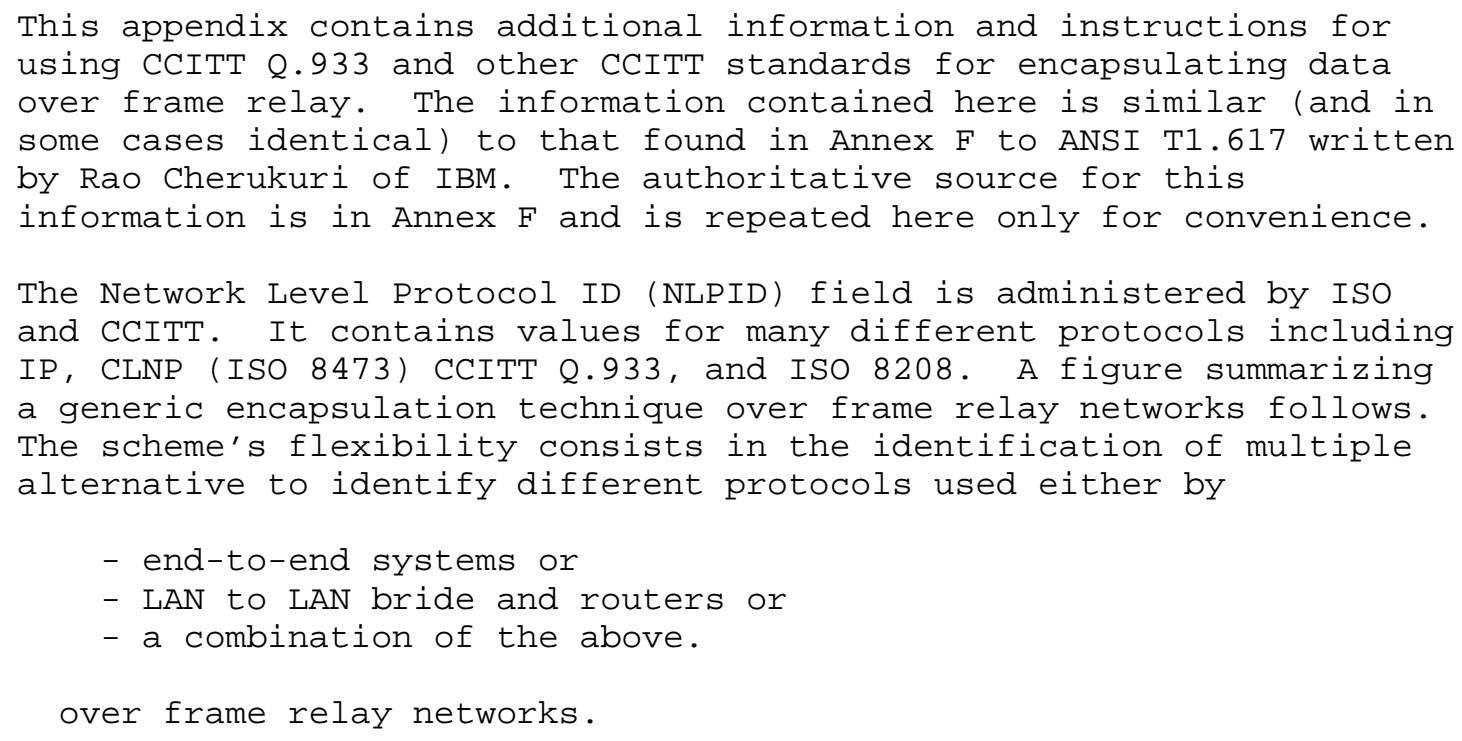




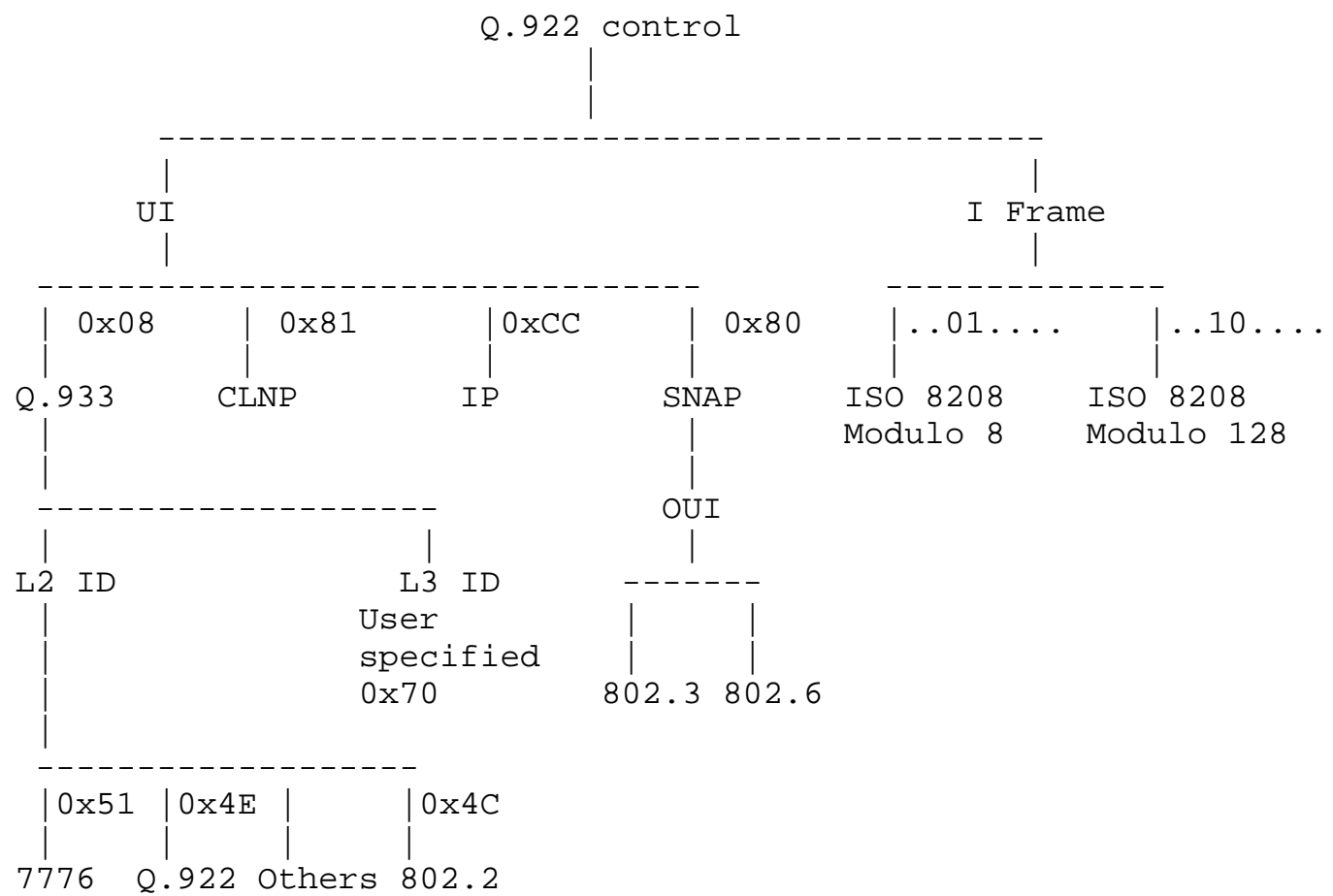

For those protocols which do not have a NLPID assigned or do not have a SNAP encapsulation, the NLPID value of 0x08, indicating CCITT Recommendation $\mathrm{Q} .933$ should be used. The four octets following the NLPID include both layer 2 and layer 3 protocol identification. The code points for most protocols are currently defined in ANSI T1.617 low layer compatibility information element. There is also an escape for defining non-standard protocols. 
Format of Other Protocols using $Q .933$ NLPID

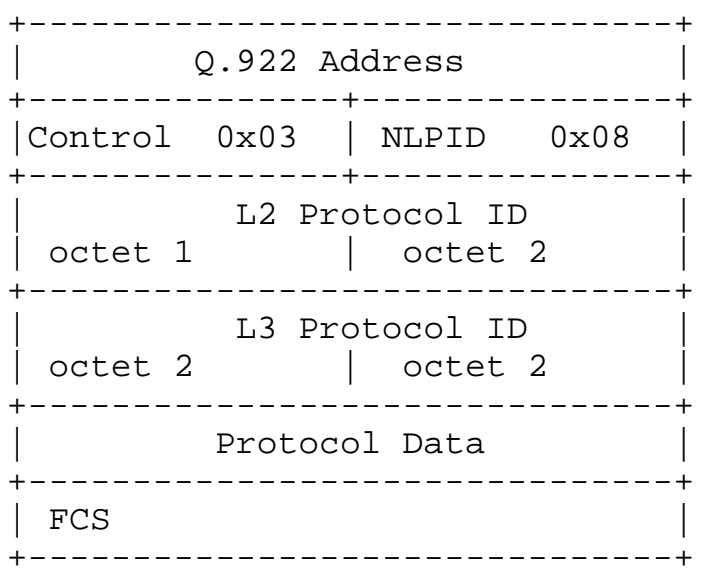

ISO $8802 / 2$ with user specified layer 3

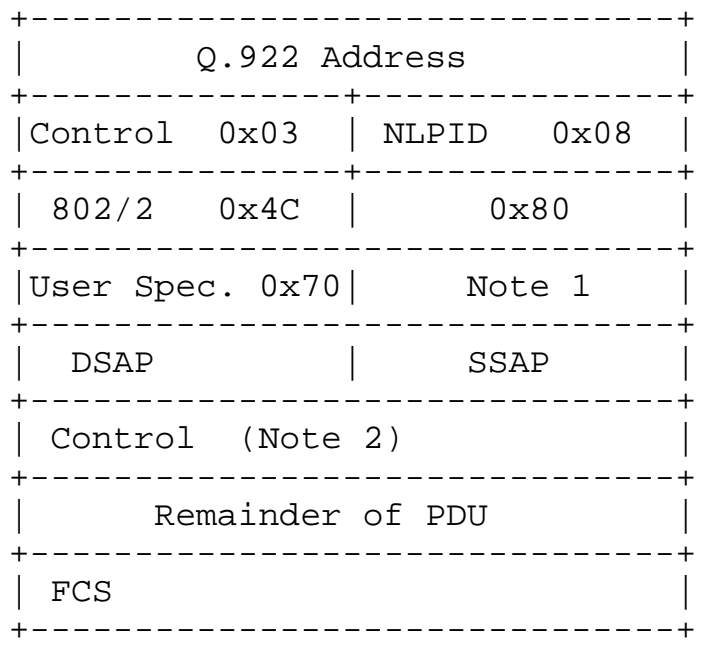

Note 1: Indicates the code point for user specified layer 3 protocol.

Note 2: Control field is two octets for I-format and S-format frames (see 88002/2)

Encapsulations using I frame (layer 2) 
The Q.922 I frame is for supporting layer 3 protocols which require acknowledged data link layer (e.g., ISO 8208). The C/R bit (T1.618 address) will be used for command and response indications.

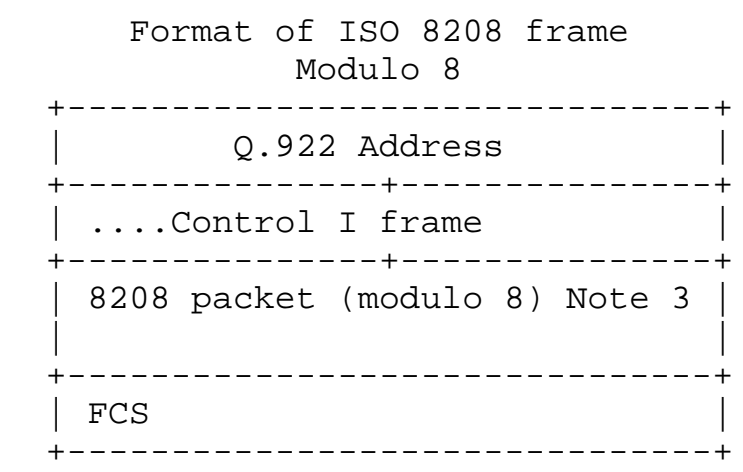
Note 3: First octet of 8208 packet also identifies the
NLPID which is "..01...".

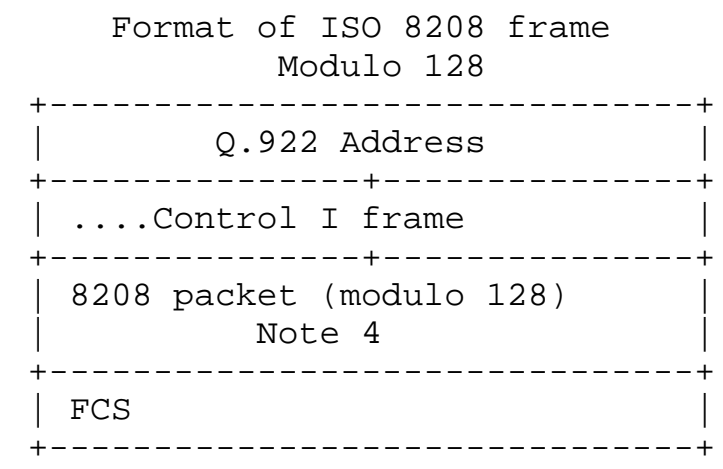

Note 4: First octet of 8208 packet also identifies the NLPID which is "..10...".

13. References

[1] International Telegraph and Telephone Consultative Committee, "ISDN Data Link Layer Specification for Frame Mode Bearer Services", CCITT Recommendation Q.922, 19 April 1991.

[2] American National Standard For Telecommunications - Integrated Services Digital Network - Core Aspects of Frame Protocol for Use with Frame Relay Bearer Service, ANSI T1.618-1991, 18 June 1991. 
[3] Information technology - Telecommunications and Information Exchange between systems - Protocol Identification in the Network Layer, ISO/IEC TR 9577: 1990 (E) 1990-10-15.

[4] Baker, F., Editor, "Point to Point Protocol Extensions for Bridging", RFC 1220, ACC, April 1991.

[5] International Standard, Information Processing Systems - Local Area Networks - Logical Link Control, ISO 8802-2: 1989 (E), IEEE Std 802.2-1989, 1989-12-31.

[6] Plummer, D., "An Ethernet Address Resolution Protocol - or Converting Network Protocol Addresses to 48.bit Ethernet Address for Transmission on Ethernet Hardware", STD 37, RFC 826, MIT, November 1982 .

[7] Reynolds, J. and J. Postel, "Assigned Numbers", STD 2, RFC 1340, USC/Information Sciences Institute, July 1992.

[8] Finlayson, R., Mann, R., Mogul, J., and M. Theimer, "A Reverse Address Resolution Protocol", STD 38, RFC 903, Stanford University, June 1984 .

[9] Postel, J. and Reynolds, J., "A Standard for the Transmission of IP Datagrams over IEEE 802 Networks", RFC 1042, USC/Information Sciences Institute, February 1988.

[10] IEEE, "IEEE Standard for Local and Metropolitan Area Networks: Overview and architecture", IEEE Standards 802-1990.

[11] Bradley, T., and C. Brown, "Inverse Address Resolution Protocol", RFC 1293, Wellfleet Communications, Inc., January 1992.

[12] IEEE, "IEEE Standard for Local and Metropolitan Networks: Media Access Control (MAC) Bridges", IEEE Standard 802.1D-1990.

[13] PROJECT 802 - LOCAL AND METROPOLITAN AREA NETWORKS, Draft Standard 802.1G: Remote MAC Bridging, Draft 6, October 12, 1992.

14. Security Considerations

Security issues are not discussed in this memo. 
15. Authors' Addresses

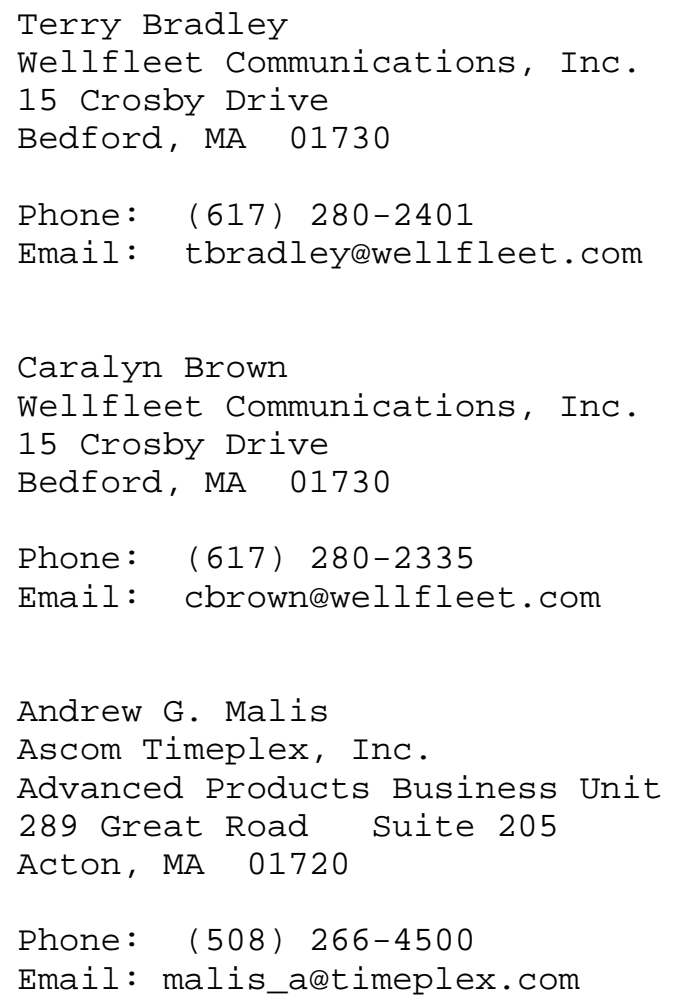

